\title{
Mesoporous aluminosilicate nanofibers with a low Si/Al ratio as acidic catalyst for hydrodeoxygenation of phenol
}

\author{
Tommy Haynes ${ }^{[a]}$, Thibaut D'hondt ${ }^{[b]}$, Alexander L. Morritt ${ }^{[c]}$, Yaroslav Z. Khimyak ${ }^{[c]}$, Didier Desmecht ${ }^{[b]}$, \\ Vincent Dubois ${ }^{[\mathrm{b}]}$ and Sophie Hermans ${ }^{*[a]}$
}

[a] IMCN Institute, MOST division, Université catholique de Louvain, 1 Place Louis Pasteur, B-1348 Louvain-la-Neuve, Belgium.

[b] Department of Physical Chemistry and Catalysis, LABIRIS, 1 Avenue Emile Gryson, B-1070 Brussels, Belgium.

[c] School of Pharmacy, University of East Anglia, Norwich Research Park, Norwich NR4 7TJ, United Kingdom.

* Corresponding author. Tel.: +32 10 472810; Fax: (+32) 10472330

E-mail: sophie.hermans@uclouvain.be

\begin{abstract}
Mesoporous aluminosilicate nanofibers (mASNF) were prepared using hard and soft dual templates approach. The mesoporous material was fully characterized and its acidic nature was confirmed by FTIR spectroscopy of pyridine adsorption and ${ }^{27} \mathrm{Al} /{ }^{29} \mathrm{Si}$ solid state NMR. Thanks to the incorporated aluminum atoms, the acidic material showed high hydrothermal stability which is an essential property for biomass conversion applications. The catalytic performance of $\mathrm{Pd}$ supported on mASNF for hydrodeoxygenation (HDO) of lignin model compound was also investigated. A complete conversion and a high selectivity towards cyclohexane (up to $95 \%$ ) starting from phenol were achieved with this bifunctional catalyst. In comparison, no cyclohexane has been produced with a non-acidic material which underlines the importance of acidic sites in HDO process selectivity control. Moreover, the catalyst can be recycled without losing its initial structure.
\end{abstract}

\section{Introduction}

The current shift from non-renewable first generation fossil fuels (coal, petroleum and natural gas) to sustainable alternatives has attracted global attention. Second generation fuels involving edible feedstocks such as corn lead unfortunately to fuel/food industries competition which is not acceptable. Third generation

[a] Mr. T. Haynes; Prof. S. Hermans IMCN Institute, MOST division

Université catholique de Louvain

1 Place Louis Pasteur, B-1348 Louvain-la-Neuve, Belgium E-mail: sophie.hermans@uclouvain.be

[b] Mr. T. D'hondt; Mr. D. Desmecht; Dr. V. Dubois Department of Physical Chemistry and Catalysis LABIRIS

1 Avenue Emile Gryson, B-1070 Brussels, Belgium

[c] Mr. A. L. Morritt; Prof. Y. Z. Khimyak School of Pharmacy

University of East Anglia

Norwich Research Park, Norwich NR4 7TJ, United Kingdom

Supporting information for this article is given via a link at the end of the document. fuels based on non-edible biomass renewable sources (lignocellulosic biomass, vegetable oils...) represent therefore an attractive option ${ }^{[1-3]}$. Before its use as biofuel, biomass is firstly converted into bio-oil via biochemical (fermentation, anaerobic digestion...) and thermochemical (gasification, liquefaction, pyrolysis...) processes ${ }^{[4,5]}$. These bio-oils are usually presented as dark brown organic liquids containing a high amount of oxygenated compounds. Although bio-oils could be considered as an alternative to petroleum-based sources, unfavorable characteristics such as high viscosity, thermal instability, and easiness of repolymerization make their use as fuel very constraining. Upgrading of bio-oils is therefore an unavoidable step in the biofuel production ${ }^{[6]}$. Two main catalytic routes are well-known to remove partially or totally oxygen atoms in bio-oils: catalytic cracking and catalytic hydrotreating. Catalytic cracking is a thermal conversion method $\left(>350^{\circ} \mathrm{C}\right)$. This process is a costeffective upgrading method because it proceeds at atmospheric pressure without any additional reactive gas. Nevertheless, some drawbacks like poor hydrocarbons yield, coking and high content of phenolic compounds in the obtained biofuel make this approach non optimal. Catalytic hydrotreating is a modified catalytic cracking method using hydrogen high pressure to remove sulfur, oxygen and nitrogen ${ }^{[7]}$. In the past, this catalytic route received little attention as petroleum contains only $1.8 \mathrm{wt} \%$ sulfur and $0.1 \mathrm{wt} \%$ oxygen. Since bio-oils obtained from biomass contain up to $35 \mathrm{wt} \%$ oxygen, hydrodeoxygenation process (HDO) and the development of novel catalysts gained considerable importance in the last few years.

The traditional industrial catalysts used for petroleum hydrodesulfurization and hydrodenitrogenation are sulfided CoMo- and NiMo-based catalysts ${ }^{[8,9]}$. These catalysts have been naturally applied in HDO of bio-oils. However, they are unstable in HDO conditions and suffer from desulfurization with time leading to sulfur enrichment of the product and catalyst deactivation ${ }^{[10]}$. To overcome this drawback, HDO catalysis has been recently oriented towards noble metal catalysts ${ }^{[11-26]}$ which are very effective to activate and cleave hydrogen molecules ${ }^{[12]}$. It is well known that the support materials have also a significant 
impact on the catalyst activity in HDO reactions ${ }^{[27-29]}$. This is due to the fact that HDO requires acid sites as well as active hydrogenation sites. The appropriate support acidity is essential to ensure good performance in hydrodeoxygenation of high oxygen content compounds. Among all heterogeneous catalysis supports, zeolites seem to be good candidate for hydroprocessing reactions due to their large surface area, high thermal/hydrothermal robustness and strong acidity (Lewis and Brönsted acid sites). However, their microporous framework is a characteristic limiting their use in reactions implying molecules larger than the zeolite's pore size. For instance, Zeng et al. have shown that the micropore size of zeolites do not allow full access to Brönsted strong acid sites resulting in low activity for deoxygenation reactions ${ }^{[30]}$. Mesoporous molecular materials (M41S) have been widely applied in many research areas thanks to their large specific surface area and their narrow pore size distribution in the mesoporous range. Unfortunately, ordered mesoporous materials present poor hydrothermal stability ${ }^{[31]}$. Hydrothermal conditions are frequently applied during biomass conversion making M41S materials unsuitable for this kind of application. A particular attention has thus been devoted to the production of mesoporous materials with high specific surface area, well-defined pore structure, high hydrothermal stability and strong acidity. It is well known that tetrahedrally coordinated trivalent aluminum atoms confer Brönsted and Lewis acidity and improve the hydrothermal stability of mesoporous silica materials ${ }^{[32]}$. Various routes have therefore been developed to incorporate aluminum atoms in M41S frameworks such as direct synthesis with two precursors ( $\mathrm{Si}$ and $\mathrm{Al})^{[33-36]}$ or post-synthesis grafting ${ }^{[37,38]}$. Nevertheless, the hydrolysis and condensation rates of silica and aluminum precursors being quite different, the synthesis of mesoporous aluminosilicate with low $\mathrm{Si} / \mathrm{Al}$ ratio is still challenging.

In this context, we propose a methodology based on the coverage of carbon nanofibers (CNF), used as 'hard' templates, by a mesoporous aluminosilicate layer using a single source $\mathrm{Al} / \mathrm{Si}$ molecular precursor, namely bis(secbutoxy)aluminoxytriethoxysilane $\left[(\mathrm{sec}-\mathrm{BuO})_{2} \mathrm{AlOSi}(\mathrm{OEt})_{3}\right]^{[39]}$. This methodology allows producing mesoporous aluminosilicate nanofibers (mASNF) with a low Si/Al ratio and with a high specific surface area. The mesoporosity is conferred by 'soft' templating approached and the carbon core is calcined to liberate the inner cavity and increased accessibility to all acidic sites in the nanostructured material. After palladium nanoparticles deposition and detailed characterization, the bifunctional catalyst obtained ( $\mathrm{Pd} / \mathrm{mASNF}$ ) will be tested for the HDO of phenol, a modelcompound representative of lignin-derived chemicals. Indeed, the valorization of lignin by converting it into fuels ${ }^{[2,11,40,41]}$ (via HDO of phenolic monomers) and/or chemicals is an economic priority within a sustainable processes framework ${ }^{[42,43]}$. The present study is focused on the selective conversion of phenol into a less corrosive and higher heating value product: cyclohexane. Moreover, the hydrothermal stability of our material will also be investigated because it is a crucial property for catalysts applied to biomass conversions in aqueous media.

\section{Results and Discussion}

In this work, mesoporous aluminosilicate nanofibers have been prepared by hard and soft dual template approach (Scheme 1). In the first step of acidic material preparation, the hard template (carbonaceous nanofibers CNF) was covered by an aluminosilicate layer. Ionic surfactant CTAB (in blue) was used as soft template to create the mesoporosity in the layer. After a calcination step to remove both the carbon core and the soft surfactant, mesoporous aluminoslicate nanofibers were obtained, that present an empty core. These have been fully characterized as described in the following sections.

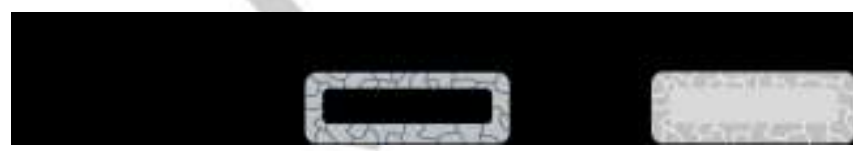

Scheme 1. mASNF preparation by hard (black) and soft (blue) dual template approach.

The XPS atomic percentages of C1s, O1s, N1s, Si2p, and Al2p after each synthesis step are given in Table 1. As expected, the starting CNF support exhibits a high level of oxygen which increases after anchoring of APTES moiety. Similarly, the atomic percentages of silicon and nitrogen increase in CNF-APTES, corroborating the amide bond formation at the CNF surface during the first step. The coverage of CNF by the mesoporous aluminosilicate layer (CNF@mAS) is confirmed by the drop in carbon atomic percentage which is inversely proportional to the raise of oxygen atomic percentage. Moreover we have observed the emergence of silicon and aluminum peaks with a high Si/Al atomic ratio of 1.2. This is attributed to the use of a single molecular precursor $(\mathrm{sec}-\mathrm{BuO})_{2} \mathrm{AIOSi}(\mathrm{OEt})_{3} \quad$ (BATEOS) presenting both $\mathrm{Al}$ and $\mathrm{Si}$ in close contact. Very few aluminosilicate materials synthesized from this mixed precursor have been reported in literature. More importantly, no material with tubular mesoporous morphology has ever been synthesized from it. Li et al. synthetized mesoporous aluminosilicate materials with Si/Al ratios from 1.0 to 10 by using the single molecular precursor BATEOS ${ }^{[39]}$. In their paper, aluminum elemental analysis showed that the content of aluminum in the final materials is almost the same as in the initial gel mixture. Shortly after, Su et al. prepared mesoporous aluminosilicates with the same precursor ${ }^{[44]}$. Low Si/Al ratios of 1.2 and 1.9 were obtained which is close to the desired value of 1 . These results prove that aluminum can be stoichiometrically incorporated into a mesoporous silica matrix by using the BATEOS precursor. To the best of our knowledge, very few studies have successfully prepared stable mesoporous materials with a low Si/AI ratio from distinguished silica and alumina sources. One well-known example is the work of Amoros et al. ${ }^{[45]}$. In this case, aluminumrich mesoporous materials of the MCM- 41 type have been synthesized using $\mathrm{Al}(\mathrm{OBu})_{3}$ and TEOS precursors in the presence of triethanolamine. However, the obtained materials present a small pore size which makes interchannel transportation of guest molecules ineffective. In our case, the Si/Al ratio is slightly higher 
than the expected value of 1 due to the presence of silicon in APTES moiety. After calcination of carbonaceous support in the core under air at $800{ }^{\circ} \mathrm{C}$ we noticed the persistence of $\mathrm{N} 1 \mathrm{~s}$ and $\mathrm{C} 1 \mathrm{~s}$ peaks. Nevertheless, the carbon/nitrogen atomic percentage has strongly diminished (Table 1) meaning that both the hard and soft templates have been removed and the mesoporous aluminosilicate nanofibers (mASNF) produced are quite pure. This result has been confirmed by elemental analysis by ICP. As shown in Table 2 the weight percentage of nitrogen and carbon in mASNF sample are below $0.1 \%$. The ca. $7 \%$ carbon detected by XPS on the surface (but not in such high amount in the bulk by ICP) are the unavoidable contamination by adventitious carbon. Moreover, the Si/Al ratio is unchanged after the calcination step (visible by XPS in Table 1 and elemental analysis in Table 2) reflecting the high thermal stability of our material. As expected, we also observed lower $\mathrm{O} / \mathrm{Si}$ and $\mathrm{O} / \mathrm{Al}$ ratios after calcination due to the removal of oxygen from hard CNF template. Another calcination temperature $\left(600^{\circ} \mathrm{C}\right)$ and longer duration time $(48 \mathrm{~h})$ have been tested. The XPS results obtained are very similar to those for the materials after the $800{ }^{\circ} \mathrm{C}$ treatment (Table S1). Therefore, the chosen optimal protocol is the calcination step at $800{ }^{\circ} \mathrm{C}$ for $24 \mathrm{~h}$.

Table 1. XPS analyses of mASNF material and bifunctional catalysts (in atomic percentage) after each synthesis step.

\begin{tabular}{|c|c|c|c|c|c|}
\hline & $\mathrm{CNF}$ & $\begin{array}{l}\text { CNF- } \\
\text { APTES }\end{array}$ & $\begin{array}{c}\mathrm{CNF@mA} \\
\mathrm{S}\end{array}$ & $\begin{array}{c}\mathrm{mASNF} \\
800^{\circ} \mathrm{C} \\
24 \mathrm{~h}\end{array}$ & $\begin{array}{l}\mathrm{Pd} / \mathrm{mASN} \\
\mathrm{F}(2 \mathrm{wt} . \%)\end{array}$ \\
\hline C1s & 94.61 & 88.45 & 35.04 & 7.05 & 7.30 \\
\hline O1s & 5.28 & 7.83 & 46.76 & 60.64 & 63.38 \\
\hline N1s & 0.07 & 1.31 & 1.25 & 0.44 & 0.20 \\
\hline $\mathrm{Cl} 2 \mathrm{p}$ & 0.04 & 0.34 & I & & 0.07 \\
\hline Si2p & I & 2.07 & 9.17 & 17.52 & 15.81 \\
\hline $\mathrm{Al} 2 \mathrm{p}$ & I & I & 7.77 & 14.35 & 13.16 \\
\hline$P d 3 d$ & I & I & I & I & 0.08 \\
\hline \multirow[t]{2}{*}{$\mathrm{Si} / \mathrm{Al}$} & I & I & 1.2 & 1.2 & 1.2 \\
\hline & $\mathrm{C}$ & $\mathrm{H}$ & $\mathrm{Si}$ & $\mathrm{Al}$ & $\begin{array}{l}\text { Si/Al } \\
\text { (mola } \\
r \\
\text { ratio) }\end{array}$ \\
\hline mASNF & $\begin{array}{l}<0.1 \\
0\end{array}$ & $<0.10$ & $\begin{array}{ll}<0.1 & 22.20 \\
0 & \end{array}$ & 18.71 & 1.1 \\
\hline $\begin{array}{l}\mathrm{Pd} / \mathrm{mASN} \\
\mathrm{F}\end{array}$ & 0.12 & 0.82 & $\begin{array}{l}<0.1 \\
0\end{array}$ & 18.50 & $\begin{array}{l}2.0 \\
2\end{array}$ \\
\hline
\end{tabular}

Transmission electron microscopy (TEM) images of CNF (a), CNF@mAS (b) and mASNF (c) are reported in Figure 1. As can be seen, an aluminosilicate layer has been uniformly deposited around the carbon nanofibers support (Figure 1b). Despite the layer thickness is quite constant all along the wall of one carbon nanofiber, it varies from one nanofiber to another. This can be explained by the fact that the starting CNFs have different diameters and surface structures which could affect the aluminosilicate deposition (Figure S1 in Electronic Supplementary Information, ESI).

It is also well known that the hydrolysis/condensation reaction rates are very different for alumina and silica precursors leading potentially to domains richer in silicon or aluminum during the aluminosilicate production. In order to confirm that the aluminosilicate layer composition is homogeneous for each covered carbon nanofiber, EDX-SEM analyses have been performed on CNF@mAS sample at seven different positions on different fibers (Figure S2). As shown in the ESI the atomic percentages of $\mathrm{C}, \mathrm{O}, \mathrm{Al}$ and $\mathrm{Si}$ are quite different from spot to spot, due to the difference in layer thickness. Nevertheless the Si/A ratios obtained at each position are very similar with a mean value of $1.12 \pm 0.05$, meaning that the aluminosilicate composition is the same for the entire sample. This result clearly proves that the use of BATEOS as single molecular precursor allows the deposition of a homogeneous aluminosilicate layer. After the calcination step at $800^{\circ} \mathrm{C}$ under air we noticed that the mASNF material (Figure 1c) maintained the carbon nanofibers tubular morphology and displayed a high degree of porosity (see also $\mathrm{N}_{2}$ physisorption analyses below). Moreover, we also observe the total elimination of CNF template after the calcination step, which agrees closely with the XPS results.

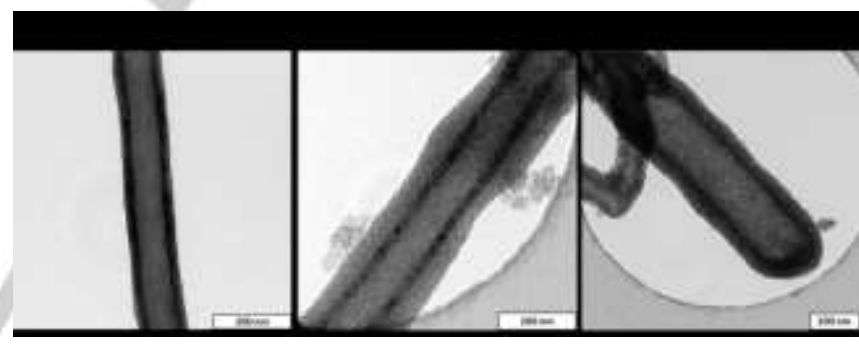

Figure 1. TEM micrographs of (a) starting CNF (b) CNF@mAS and (c) mASNF (calcined at $800^{\circ} \mathrm{C}$ ).

Figure 2a displays the nitrogen adsorption-desorption isotherms and pore size distributions obtained by the BJH method for CNF (dashed line), CNF@mAS (dotted line) and mASNF (solid line). The isotherm obtained for CNF is characteristic of a non-porous material with a low specific surface area of $26 \mathrm{~m}^{2} / \mathrm{g}$. The CNF@mAS and mASNF samples exhibit a type IV curve with $\mathrm{H} 2$ hysteresis loop which is typical of wormhole-like structure of MSUtype materials ${ }^{[39]}$. The specific surface areas developed by both materials are respectively $302 \mathrm{~m}^{2} / \mathrm{g}$ and $425 \mathrm{~m}^{2} / \mathrm{g}$ with uniform pore size distributions focused at $3.8 \mathrm{~nm}$ (Figure $2 \mathrm{~b}$ ). The Si-O and $\mathrm{Al}-\mathrm{O}$ bond lengths being different, a multimodal pore size distribution should be observed in the case of a non-homogenous aluminosilicate layer. Therefore this pore size distribution confirms the homogeneity in composition deduced from EDXSEM analyses. After the combustion of carbon nanofibers template, the increase in specific surface area is due to a combination of factors: surfactant removal, gain of nanofiber internal surface, release of pores connected to the nanofiber 
center and nanofibers opening. As we can also observe there is no substantial change in the curves shape and in the pore size distribution after calcination at $800{ }^{\circ} \mathrm{C}$ (Figure 2a). This means that the mesoporous aluminosilicate nanofibers have retained the same structure as before the calcination. These results are therefore consistent with the TEM micrographs.
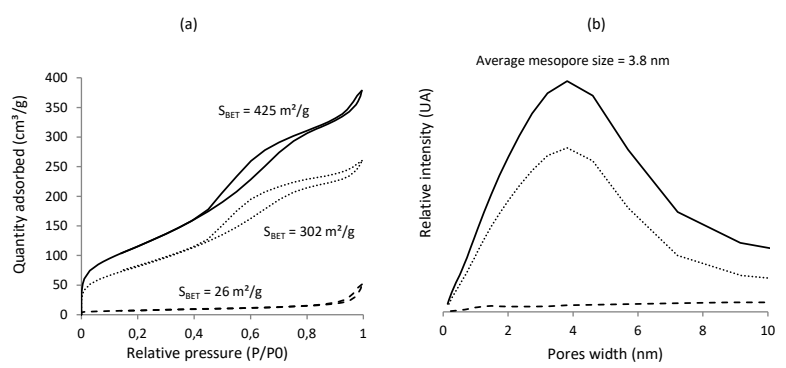

Figure 2. (a) nitrogen adsorption-desorption isotherms at $77 \mathrm{~K}$ and (b) pore size distribution obtained by the Barrett-Joyner-Halenda method for (---) CNF, (...) $\mathrm{CNF@mAS} \mathrm{and}(-)$ calcined material mASNF.

To evaluate the type of acidic sites in mASNF, FTIR spectroscopy of pyridine adsorption was carried out on the as-obtained material. The characteristic bands of pyridine appear in the FT-IR spectrum in the range of $1400-1700 \mathrm{~cm}^{-1}$. Figure S3 in ESI shows the spectrum of pyridine adsorbed on mASNF treated at $150{ }^{\circ} \mathrm{C}$. As expected the sample gives bands attributed to pyridine adsorbed on Brönsted acid sites (1547 and $1639 \mathrm{~cm}^{-1}$ ), on Lewis acid sites (1456 and $1622 \mathrm{~cm}^{-1}$ ) and a band at $1491 \mathrm{~cm}^{-1}$ corresponding to both Lewis and Brönsted acid sites. The concentration of Lewis and Brönsted acid sites and the ratio L/B have been calculated (Table S2) as reported in literature ${ }^{[46]}$. As expected the incorporation of aluminium atoms in the silica framework confers acidity to the material: $0.73 \mathrm{mmol} / \mathrm{g}$ total acidity, for $0.47 \mathrm{mmol} / \mathrm{g}$ Brönsted acid sites and $0.26 \mathrm{mmol} / \mathrm{g}$ Lewis acid sites. The acid sites concentration is comparable to that reported in the study using the same precursor ${ }^{[39]}$. Nevertheless, the ratio of Lewis acid sites versus Brönsted acid sites (L/B ratio) is quite different with a much higher Brönsted acidity in our case. Incorporation of Aluminum also has a notable impact on the mesoscopic ordering of mesoporous silica materials ${ }^{[39,46]}$. As shown in the X-ray diffractograms (Figure S4) mASNF exhibits a broader diffraction peak at low $2 \theta$ value in comparison with mesoporous silica nanofibers (mSNF). This result clearly indicates that a high aluminum content decreases the structural order of the material.

${ }^{29} \mathrm{Si}$ and ${ }^{27} \mathrm{Al}$ solid-state NMR spectra of CNF@mAS material are consistent with typical spectra for zeolites and aluminosilicates with high Al/Si ratio (Figure 3). The ${ }^{27} \mathrm{Al}$ MAS NMR spectra show two resonances attributable to $\mathrm{AI}(\mathrm{IV})(58.2 \mathrm{ppm})$ and $\mathrm{AI}(\mathrm{VI})(4.8$ $\mathrm{ppm})$. High population of $\mathrm{Al}(\mathrm{VI})$ sites is consistent with the presence of relatively large amount of extra-framework $\mathrm{Al}^{[47]}$ while tetrahedrally coordinated aluminum atoms bring acidity to the material. The ${ }^{29} \mathrm{Si}$ NMR spectrum shows two broad lines attributable to ${ }^{4} \mathrm{Q} \mathrm{Si}$ sites with different number of $\mathrm{Al}(\mathrm{IV})$ in the coordination sphere (i.e. $\mathrm{Si}(\mathrm{OSi})_{4-\mathrm{n}}(\mathrm{OAI})_{\mathrm{n}}, \mathrm{n}=1,2$ based on the values of $\left.{ }^{29} \mathrm{Si}\right)^{[48]}$. One could also assume the presence of some ${ }^{3} \mathrm{Q}$ sites population (i.e. $\left.\mathrm{Si}(\mathrm{OSi})_{3-\mathrm{n}}(\mathrm{OAI})_{\mathrm{n}}(\mathrm{OH}), \mathrm{n}<2\right)$. These are transformed into ${ }^{4} Q$ sites upon thermal treatment, when comparing with the spectra for mASNF. Indeed, the ${ }^{29} \mathrm{Si}$ MAS NMR spectrum of calcined mASNF material shows a very broad line centred at ca. $-101 \mathrm{ppm}$ with apparent reduced population of the sites at ca. $-92 \mathrm{ppm}$. The thermal treatment resulting in the formation of mASNF had a dramatic effect on the ${ }^{27} \mathrm{Al}$ MAS NMR spectrum which now displays three sites, consistent with AI(IV) (59.3 ppm), $\mathrm{Al}(\mathrm{V})$ (31.7 ppm) and $\mathrm{Al}(\mathrm{VI})$ (3.7 ppm) sites. The assignment of the peak at $31.7 \mathrm{ppm}$ to pentahedral $\mathrm{Al}$ is in line with the literature reporting on the thermal treatment of zeolites and aluminosilicates ${ }^{[46,47]}$.

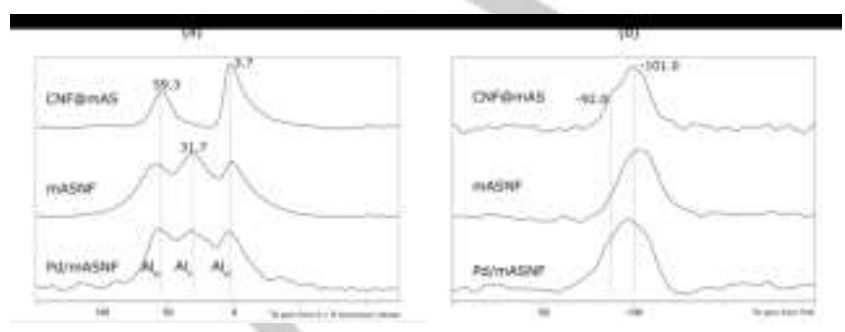

Figure 3. : (a) ${ }^{27} \mathrm{Al}$ MAS NMR of CNF@mAS (top), mASNF (middle) and $\mathrm{Pd} / \mathrm{mASNF}$, (b) ${ }^{29} \mathrm{Si}$ MAS NMR of CNF@mAS (top), mASNF (middle) and $\mathrm{Pd} / \mathrm{mASNF}$

Hydrothermal stability is one of the most important properties required for porous aluminosilicate. Many applications, in particular in the field of biomass conversion, are carried out in aqueous media or in the presence of water ${ }^{[49]}$. Moreover, the high content of water (above $50 \mathrm{wt} . \%$ ) in raw bio-oil and its formation during HDO process are two additional factors to take into account ${ }^{[50]}$. It is well known that pure mesoporous silica such as MCM-41 and MCM-48 are weakly resistant to harsh hydrothermal conditions $^{[31,51]}$. To increase their hydrothermal stability, surface modifications with hydrophobic moieties ${ }^{[52-54]}$ or aluminium species are often undertaken ${ }^{[32,37,55,56]}$. Therefore our mASNF material has been treated under refluxing water for $6 \mathrm{~h}$ to evaluate this property. Table 3 exhibits the atomic percentages from XPS analysis on mASNF before and after hydrothermal treatment. The $\mathrm{Si} / \mathrm{Al}$ ratio decreases slightly after hot water treatment due to the removal of $\mathrm{Si}$ atom (from 17.52 at.\% to 16.51 at.\%). Consequently the amount of oxygen decreases and carbon increases. This slight loss of silica does not appear to be sufficient to affect the mASNF structure as shown in the TEM pictures (Figure S5 in ESI). Even if we noticed some shortened nanofibers, the tubular morphology is globally maintained. The weak hydrothermal alteration of mASNF structure is also reflected in the nitrogen sorption curves shown in Figure $4 a$. The specific surface area decreased slightly from $425 \mathrm{~m}^{2} / \mathrm{g}$ to $375 \mathrm{~m}^{2} / \mathrm{g}$ but without any change in the pore size distribution (Figure 4b). Comparatively, a sample covered by a pure silica layer (before removal of the carbon core), prepared as described in our previous work [30], was treated under similar conditions. After the hot water treatment, the silica layer has been totally destroyed leaving only the bare carbon core. All these results highlight the structural stability of the produced mASNF material in hydrothermal conditions, which is a significant advantage for green chemistry applications. 
Table 3. XPS analyses of mASNF material after hydrothermal treatment.

\begin{tabular}{ccc}
\hline & mASNF & mASNF 6h $\mathrm{H}_{2} \mathrm{O}$ \\
\hline $\mathrm{C} 1 \mathrm{~s}$ & 7.05 & 9.94 \\
$\mathrm{O} 1 \mathrm{~s}$ & 60.64 & 58.54 \\
$\mathrm{~N} 1 \mathrm{~s}$ & 0.44 & 0.36 \\
$\mathrm{Cl} 2 \mathrm{p}$ & $/$ & $/$ \\
$\mathrm{Si} 2 \mathrm{p}$ & 17.52 & 16.51 \\
$\mathrm{Al} 2 \mathrm{p}$ & 14.35 & 14.65 \\
$\mathrm{Si} / \mathrm{Al}$ & 1.22 & 1.13 \\
\hline
\end{tabular}
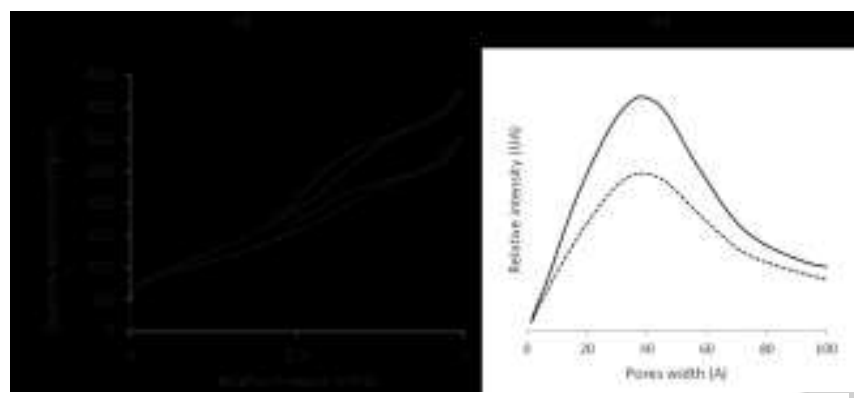

Figure 4. Nitrogen adsorption-desorption isotherms at $77 \mathrm{~K}$ for $(-) \mathrm{mASNF}$ and (...) mASNF after thermal treatment.

As mentioned in the introduction, HDO process requires both acid and hydrogenation sites ${ }^{[57]}$. Therefore, palladium nanoparticles have been deposited on the mASNF as-obtained material (synthesis procedure described in the experimental section). The bifunctional catalyst has been characterized by XPS, elemental analysis, TEM, nitrogen physisorption and ${ }^{27} \mathrm{Al} /{ }^{29} \mathrm{Si} \mathrm{NMR}$. After the nanoparticles deposition, a Pd peak with a binding energy typical of metallic palladium ${ }^{[58]}$ (335.1 eV for $\left.\mathrm{Pd}_{3 \mathrm{~d} 5 / 2}\right)$ appears in the XPS spectrum (Figure S6). The XPS results also reveal that the nanoparticles deposition has modified only slightly the surface atomic percentages (Table 1). More importantly, the Si/Al ratio does not change at all. Elemental analysis also confirmed the presence of palladium on mASNF with a loading value of $2 \mathrm{wt}$. $\%$, as expected (Table 2). It may be noted that the nanoparticles deposition does not affect the weight percentage of aluminium and silicon which is in accordance with XPS results. Moreover, the specific surface area of the bifunctional catalyst has slightly decreased $\left(398 \mathrm{~m}^{2} / \mathrm{g}\right)$ compared to the bare support, with a lower average mesopore size $(3.3 \mathrm{~nm})$. The incorporation of $\mathrm{Pd}$ has little effect on the ${ }^{29} \mathrm{Si}$ and ${ }^{27} \mathrm{Al}$ MAS NMR spectra (see Figure 3 ). Most importantly, the $\mathrm{Pd} /-\mathrm{mASNF}$ still exhibits a considerable population of $\mathrm{Al}(\mathrm{IV})$ sites which are most likely responsible for its Brönsted acidity ${ }^{[46]}$. Finally, TEM micrographs (Figure S7) showed that palladium nanoparticles are uniformly deposited on mASNF, with a size of ca $5 \mathrm{~nm}$.

The catalytic performance of the synthetized catalyst was evaluated in the HDO of phenol into cyclohexane. The HDO reaction can occur through different pathways (Scheme 2) depending on several different parameters and therefore a large range of products could be obtained. The conversion and the selectivity toward the predominant products are given in Table 4. To determine the activity of mASNF material and the influence of supported metallic nanoparticles, the catalytic tests were first carried out without catalyst (blank, entry 1). This test shows no conversion $(0 \%)$ meaning that the HDO process cannot occur in the absence of catalyst under the selected conditions. When the mASNF without Pd are added the conversion is unchanged (entry 2 ). Both tests were carried out at $200{ }^{\circ} \mathrm{C}$. This result is not surprising because it is well reported in the literature that HDO of phenolic species derived from lignin is quite difficult due to the strong $\mathrm{Ar}-\mathrm{O}$ bond ${ }^{[59]}$. Therefore HDO of phenol often requires firstly hydrogenation of the aromatic ring to produce cyclohexanol before getting cyclohexane (Scheme 2$)^{[60]}$. Under mild conditions (under $300{ }^{\circ} \mathrm{C}$ ) partial hydrogenation takes place and cyclohexanone is also obtained which is rapidly hydrogenated into cyclohexanol. Then deoxygenation of cyclohexanol occurs either through dehydratation to cyclohexene or hydrogenolysis to cyclohexane.

It was shown that hydrodeoxygenation of phenol is strongly affected by the temperature ${ }^{[20]}$. The influence of reaction temperature was consequently investigated with our bifunctional catalyst. A catalytic test was first carried out at $100{ }^{\circ} \mathrm{C}$, which displayed a low conversion of phenol (38\%) without any cyclohexane produced (entry 3 ). However, when the reaction is carried out at $200{ }^{\circ} \mathrm{C}$ (entry 4), the bifunctional catalyst reaches a conversion of $99 \%$ with a high selectivity towards cyclohexane (up to $95 \%$ ) in 2 hours, corroborating results reported in literature ${ }^{[20]}$. These results also show that the acidic sites of mASNF are still accessible after the nanoparticles deposition (see discussion on NMR results above). No unsaturated products like benzene have been observed which is in accordance with Jones' study[61] Moreover, the large pore size of mASNF allows a full accessibility of phenol to these acidic sites. Zeng's study demonstrated that a pore size around $3 \mathrm{~nm}$ is one of the key factors for the diffusion of phenol and ensures excellent performance in $\mathrm{HDO}^{[30]}$. In our case, the $\mathrm{Pd} / \mathrm{mASNF}$ presents an average pore size of $3.3 \mathrm{~nm}$ which is largely sufficient for phenol transport in the mASNF porous network. In order to confirm that the selectivity towards cyclohexane is due to the acid sites of mASNF, palladium supported on pure mesoporous silica nanofibers (Pd/mSNF) was also tested in HDO of phenol. Indeed, De Souza et al. have recently shown, by $\mathrm{NH}_{3}$-TPD measurements and cyclohexanol dehydration tests, that palladium supported on silica support exhibits a very low density of acid sites ${ }^{[27]}$. In our case, mSNF does not disclose any peak in $\mathrm{NH}_{3}$-TPD compared to mASNF material that displays a broad peak between $150-250{ }^{\circ} \mathrm{C}$ (Figure S8). These results confirm that (i) our silica based nanofibers are non-acidic materials (ii) the incorporation of aluminium atoms brings acidity. As shown in Table 4 (entry 5), the Pd/mSNF catalyst displays a very good activity thanks to palladium nanoparticles but no cyclohexane (or cyclohexene) has been obtained. In fact, it was also shown that over non-acidic materials cyclohexanol $^{[62]}$ could be rapidly produced. This result proves again the importance of strong acid sites to produce deoxygenated compounds in HDO process. Nevertheless, the amount of acid sites also plays a critical role. By introducing a lower amount of catalyst (entry 6), the selectivity towards 
cyclohexane drops drastically $(11 \%)$ indicating that a minimum of acidity is required to obtain high performance for deoxygenation reaction.

The recyclability of $\mathrm{Pd} / \mathrm{mASNF}$ catalyst was investigated. It is well known that HDO reactions produce coke, due to ring condensation at the catalyst surface, which is responsible for it deactivation ${ }^{[29,50]}$. Coking is typically favored over acidic catalysts, by low pressure and high temperature, but also in the presence of metal sites. S. Echeandia et al. claimed that the coke formation is strongly dependent on surface concentrations of acid sites: the higher the concentration, the larger coke formation ${ }^{[62]}$. It is also well known that the regeneration by coke combustion is influenced by temperature and oxygen concentration of the applied post-treatment ${ }^{[63]}$. The $\mathrm{Pd} / \mathrm{mASNF}$ was therefore regenerated after the catalytic test by treatment at $400{ }^{\circ} \mathrm{C} / 600^{\circ} \mathrm{C}$ under air for different durations. When the catalyst is reused without regeneration the complete conversion is maintained, but the selectivity has been drastically modified (Table 4 , entry 7 ), demonstrating that the acid sites are almost completely deactivated. Interestingly, the catalyst still exhibits high specific surface area $\left(386 \mathrm{~m}^{2} / \mathrm{g}\right)$ and identical pore size distribution as the fresh catalyst (Figure S9). To make sure that this loss of selectivity is due to coke deposition during the reaction, a catalytic test was performed for a longer time with a more concentrated phenol solution (entry 8 ). The phenol is still totally converted, but the selectivity towards cyclohexane is too low in comparison with the value that we should obtain (calculated from the first run (entry 4), as a function of phenol concentration and catalyst mass engaged) This result is very important because it confirms that the acid sites are deactivated during the test and not during the washing step. On the other hand, after regeneration (entry 9-11) the selectivity towards cyclohexane increases gradually with temperature/duration of treatment, meaning that the coke can be removed and therefore acid sites are accessible again. When the strongest regeneration procedure was performed (entry 11), a slight decrease of specific area was observed $\left(349 \mathrm{~m}^{2} / \mathrm{g}\right)$ with no change in the pore size distribution (Figure S9). This small loss could be explained by the formation of larger palladium nanoparticles during the regeneration step that probably block some internal channels of the aluminosilicate framework. A recent study has reported the pathways for catalyst deactivation by coke during the hydrodeoxygenation of raw bio-oil[50]. Nevertheless, the conditions that favor coke formation are numerous and depend on a lot of parameters, that have not been fully elucidated so far, which makes challenging the design of recyclable catalysts in HDO processes.

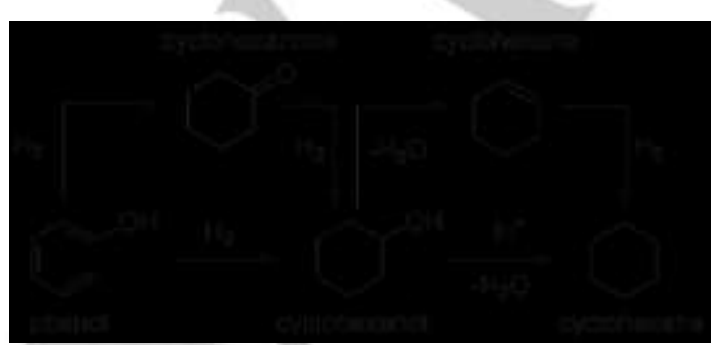

Scheme 2. General pathway for the hydrodeoxygenation of phenol over bifunctional catalysts.
Table 4. Conversion and selectivity for the HDO of phenol after $2 \mathrm{~h}$ reaction at $200{ }^{\circ} \mathrm{C}$.

\begin{tabular}{cccccc}
\hline & Catalyst & Run & $\begin{array}{c}\text { Conversion } \\
(\%)\end{array}$ & $\begin{array}{c}\text { Cyclohexanol } \\
\text { selectivity }(\%)\end{array}$ & $\begin{array}{c}\text { Cyclohexane } \\
\text { selectivity }(\%)\end{array}$ \\
\hline 1 & Blank & 1 & 0 & 0 & 0 \\
2 & mASNF & 1 & 0 & 0 & 0 \\
3 & Pd/mASNF & $1^{[a]}$ & 38 & 14 & 0 \\
4 & Pd/mASNF & 1 & $>99$ & 5 & 95 \\
5 & Pd/mSNF & 1 & $>99$ & 24 & 0 \\
6 & Pd/mASNF & $1^{[b]}$ & $>99$ & 84 & 11 \\
7 & Pd/mASNF & 2 & $>99$ & 82 & 10 \\
8 & Pd/mASNF & $1^{[c]}$ & $>99$ & 74 & 20 \\
9 & Pd/mASNF & $2^{[\mathrm{d}]}$ & $>99$ & 76 & 18 \\
10 & Pd/mASNF & $2^{[\mathrm{e}]}$ & $>99$ & 67 & 28 \\
11 & Pd/mASNF & $2^{[\mathrm{fl}}$ & $>99$ & 59 & 39 \\
\hline
\end{tabular}

[a] $100{ }^{\circ} \mathrm{C}$ instead of $200^{\circ} \mathrm{C}$. [b] $50 \mathrm{mg}$ catalyst instead of $100 \mathrm{mg}$ [c] Catalytic test conditions: $200{ }^{\circ} \mathrm{C}, 4 \mathrm{~h}, 650 \mathrm{rpm}, 40$ bars $\mathrm{H}_{2}, 100 \mathrm{mg}$ catalyst and $75 \mathrm{~mL}$ phenol $(0.350 \mathrm{~mol} / \mathrm{L})$. Regeneration conditions: [d] $3 \mathrm{~h}$ under air at $400{ }^{\circ} \mathrm{C}$, followed by $2 \mathrm{~h}$ under $\mathrm{H}_{2} / \mathrm{N}_{2}(5 / 95)$ at $300^{\circ} \mathrm{C}$. [e] $3 \mathrm{~h}$ under air at $600{ }^{\circ} \mathrm{C}$, followed by $2 \mathrm{~h}$ under $\mathrm{H}_{2} / \mathrm{N}_{2}(5 / 95)$ at $300{ }^{\circ} \mathrm{C}$. [f] $16 \mathrm{~h}$ under air at $600{ }^{\circ} \mathrm{C}$, followed by 2 h under $\mathrm{H}_{2} / \mathrm{N}_{2}(5 / 95)$ at $300^{\circ} \mathrm{C}$.

\section{Conclusions}

The production of acidic mesoporous nanofiber material (mASNF) has been successfully achieved by using a dual-templating approach. Detailed characterizations have been undertaken to demonstrate the porous and acidic nature of the nanostructured material. It has also been shown that the incorporation of high aluminum content ( $\mathrm{Si} / \mathrm{Al}$ ratio of 1.2 ), thanks to aluminosilicate ester as single source precursor, enhances the hydrothermal stability by maintaining pore size distribution and relatively high specific surface area under hot water treatment. These results were confirmed by TEM, nitrogen physisorption and XPS measurements. Moreover, the tetrahedral aluminum sites are also very stable after calcination at high temperature which is very important for catalytic applications and catalysts regeneration. Palladium nanoparticles were supported on this acidic support and the bifunctional catalyst obtained was tested in the HDO process of phenol, a lignin model compound. A total conversion and a high selectivity (up to 95\%) towards hydrodeoxygenated product, namely cyclohexane, have been obtained. These results have been attributed to the large pores and the high specific surface area of the material which allow a full accessibility to the acidic sites and the palladium nanoparticles. Furthermore the present work has underlined the importance of acid sites in HDO process under mild conditions $\left(\mathrm{T}<300{ }^{\circ} \mathrm{C}\right)$. Indeed, non-acidic material does not produce any cyclohexane. Moreover, it was shown that the material is reusable with partial recovery of initial selectivity. It is believed that this simple methodology can be 
extended to produce various stable acidic mesostructures by changing the hard and soft templates nature. The tubular morphology of our material also offers inter alia the possibility to encapsulate magnetic nanoparticles to facilitate separation from the reaction mixture by simple magnetic filtration techniques. Furthermore, the material described in the present work could be evaluated in other HDO processes.

\section{Experimental Section}

\section{Reagents and Materials}

Carbon nanofibers (CNF, type Pyrograf III PR-24-XT-LHT-OX) were supplied by Applied Sciences Inc. (USA). Hexadecyltrimethylammonium bromide (CTAB, 95\%), (3aminopropyl) triethoxysilane (APTES, 99\%), sodium tetrachloropalladate (II) $\left(\mathrm{Na}_{2} \mathrm{PdCl}_{4}, 98 \%\right)$, and thionyl chloride $\left(\mathrm{SOCl}_{2},>99 \%\right)$ were purchased from Sigma Aldrich. Bis(secbutoxy)aluminoxytriethoxysilane $\quad\left[(\mathrm{sec}-\mathrm{BuO})_{2} \mathrm{AIOSi}(\mathrm{OEt})_{3}\right]$ (BATEOS) was purchased from Alfa Aesar. A commercial ultrasonic cleaner (VWR) was used for sonication.

\section{Synthesis of mesoporous aluminosilicate nanofibers (mASNF)}

The synthesis of mesoporous aluminosilicate nanofibers (mASNF) has been adapted from our previously-reported $\mathrm{CNF} @ \mathrm{mSiO}_{2}$ preparation $^{[64]}$. Namely, $2 \mathrm{~g}$ of carbon nanofibers (CNF) were introduced in a $250 \mathrm{~mL}$ round-bottom flask containing $100 \mathrm{~mL}$ of toluene. $6 \mathrm{~mL}$ of $\mathrm{SOCl}_{2}$ were added and the mixture was heated for $5 \mathrm{~h}$ under reflux $\left(120^{\circ} \mathrm{C}\right)$. Then, it was filtered out and extensively washed with toluene $(500 \mathrm{~mL})$. The resulting material $(\mathrm{CNF}-\mathrm{Cl})$ was dried overnight under vacuum at $100^{\circ} \mathrm{C} .1$ $\mathrm{g}$ of CNF-Cl was introduced in a $250 \mathrm{~mL}$ round-bottom flask containing $100 \mathrm{~mL}$ of dichloromethane. $1 \mathrm{~mL}$ of APTES was added and the mixture was stirred for $24 \mathrm{~h}$ at room temperature. The material (CNF-APTES) was filtered out, washed with dichloromethane $(250 \mathrm{~mL})$ and methanol $(250 \mathrm{~mL})$ and dried overnight under vacuum at $100{ }^{\circ} \mathrm{C}$. Then, $1 \mathrm{~mL}$ of BATEOS was introduced in a $100 \mathrm{~mL}$ round bottom flask containing $1.73 \mathrm{~g}$ of CTAB dissolved in $40 \mathrm{~mL}$ of ethanol. $250 \mathrm{mg}$ of CNF-APTES were added and the mixture was stirred $1 \mathrm{~h}$ at room temperature. To this suspension, $40 \mathrm{ml}$ of water were added dropwise within 4 hours. Then the solution was charged into a propylene bottle, which was closed tightly and heated at $100{ }^{\circ} \mathrm{C}$ for 3 days. The product was filtered out, washed with ethanol $(250 \mathrm{~mL})$ and dried at $100{ }^{\circ} \mathrm{C}$ overnight. The CTAB template was removed by refluxing in ethanol the solid material. Finally, CNF@maluminosilicate sample was placed into porcelain combustion boats and heated during a selected time at $800{ }^{\circ} \mathrm{C}$ under air to produce the mASNF material without the carbon core. During calcination residual CTAB surfactant is also removed.

\section{Synthesis of mesoporous silica nanofibers (mSNF)}

Mesoporous pure-silica nanofibers (mSNF) were also synthesized as previously described ${ }^{[64]}$, to be used as $100 \% \mathrm{SiO}_{2}$ reference.

\section{Synthesis of supported Pd catalysts (Pd/mASNF or $\mathrm{Pd} / \mathrm{mSNF}$ )}

$\mathrm{Pd}$ nanoparticles were supported on mesoporous aluminosilicate or pure-silica nanofibers as follows: $300 \mathrm{mg}$ of $\mathrm{mASNF} / \mathrm{mSNF}$ were suspended in $50 \mathrm{ml}$ of ethanol. $17 \mathrm{mg}$ of $\mathrm{Na}_{2} \mathrm{PdCl}_{4}$ (2 wt. \% $\mathrm{Pd}$ ) were added and the solution was stirred for $30 \mathrm{~min}$. Then, ethanol was removed in a rotary evaporator till dryness. Finally the samples were placed into porcelain combustion boat and heated during $2 \mathrm{~h}$ under a stream of $\mathrm{N}_{2} / \mathrm{H}_{2}(95: 5)$ at $300^{\circ} \mathrm{C}$.

\section{Instrumental}

The solid catalysts were characterized by X-ray photoelectron spectroscopy (XPS), transmission/scanning electron microscopy (TEM/SEM), FTIR spectroscopy of pyridine adsorption, ${ }^{27} \mathrm{Al}$ and ${ }^{29} \mathrm{Si}$ MAS NMR, elemental analyses (ICP), $\mathrm{N}_{2}$ physisorption, X-ray powder diffraction (XRD) and Temperature-Programmed Desorption (TPD) of ammonia.

XPS analyses were carried out at room temperature with a SSIX-probe (SSX 100/206) photoelectron spectrometer from Surface Science Instruments (USA), equipped with a monochromatized microfocus Al X-ray source. Samples were stuck onto small sample holders with double-face adhesive tape and then placed on an insulating ceramic carousel (Macor ${ }^{\circledR}$, Switzerland). Charge effects were avoided by placing a nickel grid above the samples and using a flood gun set at $8 \mathrm{eV}$. The binding energies were calculated with respect to the $\mathrm{C}-(\mathrm{C}, \mathrm{H})$ component of the $\mathrm{C} 1 \mathrm{~s}$ peak fixed at $284.8 \mathrm{eV}$. Data treatment was performed using the CasaXPS program (Casa Software Ltd., UK). The peaks were decomposed into a sum of Gaussian/Lorentzian (85/15) after subtraction of a Shirley-type baseline.

TEM images were obtained on a LEO 922 Omega Energy Filter Transmission Electron Microscope operating at $120 \mathrm{kV}$. The samples were suspended in hexane under ultrasonic treatment. A drop of the suspension was deposited on a holey carbon film supported on a copper grid (Holey Carbon Film 300 Mesh Cu, Electron Microscopy Sciences), which was dried overnight under vacuum at room temperature, before introduction in the microscope.

Acidity measurements of the catalyst surface were done by pyridine adsorption and temperature programmed desorption as follows ${ }^{[65]}$. The catalysts were pressed to obtain a wafer. The wafer was weighted and then placed in a sample holder inside a Pyrexcell especially designed for the controlled heating of the sample under vacuum and equipped with an optical $\mathrm{NaCl}$ window. The sample holder was movable and it allowed the sample placement in the infrared beam for the recording of IR spectra or in the furnace for the thermal treatments. In a typical measurement, the sample was heated at $300{ }^{\circ} \mathrm{C}$ under vacuum for $3 \mathrm{~h}$ in order to desorb physisorbed molecules from the surface. After cooling under vacuum, $1000 \mathrm{~Pa}$ of pyridine was sent at room temperature in the cell and adsorption was allowed for $30 \mathrm{~min}$. The sample was then outgassed at $150^{\circ} \mathrm{C}$ and $10-5 \mathrm{~Pa}$ pressure For the desorption step, once the value of 10-5 Pa was reached, the sample was kept under vacuum for $1 \mathrm{~h}$. FT-IR spectra were taken in transmission mode before and after pyridine adsorption and after the desorption step using a spectrometer IFS55 Equinox 
(Bruker) equipped with a DTGS detector. The spectra were recorded with 100 scans between 400 and $4000 \mathrm{~cm}^{-1}$.

${ }^{29} \mathrm{Si}$ solid-state spectra were acquired on a $300 \mathrm{MHz}$ Bruker Avance solid-state spectrometer at $59.60 \mathrm{MHz}$. Recycle delay of $120 \mathrm{~s}$ was used over $1 \mathrm{k}$ scans with a pulse width of $4.2 \mu \mathrm{sec}$ at $4 \mathrm{KHz}$ MAS rate. Line broadening of $100 \mathrm{~Hz}$ has been applied. The spectra are referenced to $0 \mathrm{ppm}$ with TMS. ${ }^{27} \mathrm{AI}$ MAS NMR spectra were acquired on a Bruker $400 \mathrm{MHz}$ Bruker Avance III solid state Spectrometer at $104.26 \mathrm{MHz}$. The spectra are referenced to 0 ppm with $\mathrm{AINO}_{3} 0.1 \mathrm{M}$ static sample. A recycle delay of 2 seconds was used over $2 \mathrm{k}$ scans with a pulse width of $0.33 \mu \mathrm{s}$ ( $\pi / 12$ pulse) at $10 \mathrm{KHz}$ MAS rate.

The elemental analyses $(\mathrm{C}, \mathrm{H}, \mathrm{N}, \mathrm{Si}, \mathrm{Al}, \mathrm{Ni}, \mathrm{Au}, \mathrm{Pd})$ were carried out by MEDAC Ltd., UK by microgravimetry for $\mathrm{C}, \mathrm{H}, \mathrm{N}, \mathrm{O}$ (direct measure) and by ICP after acid digestion for $\mathrm{Al}, \mathrm{Si}, \mathrm{Ni}, \mathrm{Au}$ and $\mathrm{Pd}$.

The pore texture of the covered catalysts was characterized by nitrogen adsorption-desorption isotherms. The measures were achieved by using a Micromeritics ASAP 2020 analyzer at $77 \mathrm{~K}$. Before analysis, the samples $(0.02-0.10 \mathrm{~g})$ were degassed for 2 h at $473 \mathrm{~K}$ with a heating rate of $10 \mathrm{~K} / \mathrm{min}$ under $0.133 \mathrm{~Pa}$ pressure. The analysis of the isotherms provided specific surface areas calculated with the Brunauer-Emmett-Teller (BET) equation, $\mathrm{S}_{\mathrm{BET}}$. The pore volume, $\mathrm{Vp}$, of the samples and the pores average diameter were calculated using the Barrett-Joyner-Halenda (BJH) model.

XRD experiments were carried out on a D8 Advanced diffractometer from Bruker. Each diffractogram was recorded using a linkeye XE-T detector (Bruker) in the $0.8-10^{\circ}$ (2 Theta) range with an increment of $0.02^{\circ}$ and an integration time of $0.4 \mathrm{~s}$. Eva software was used for data treatment.

TPD- $\mathrm{NH}_{3}$ analyses were performed on Hiden Catlab reactor combined with a QGA Hiden quadrupole mass spectrometer. Samples were pretreated under $\operatorname{Ar}(30 \mathrm{ml} / \mathrm{min}, 5.0$ AirLiquide $)$ at $200{ }^{\circ} \mathrm{C}$ during two hours. $\mathrm{NH}_{3}$ adsorption was performed at $70{ }^{\circ} \mathrm{C}$ during 1 hour by flowing a mixture of Argon $(20 \mathrm{ml} / \mathrm{min})$ and $5 \%$ $\mathrm{NH}_{3}$ in $\mathrm{He}(10 \mathrm{ml} / \mathrm{min})$. The catalyst was flushed in $\operatorname{Ar}(30 \mathrm{ml} / \mathrm{min})$ during 2 hours and then, the $\mathrm{NH}_{3}$ desorption measurement was performed under $\operatorname{Ar}\left(30 \mathrm{ml} / \mathrm{min}\right.$ ) from 70 to $600{ }^{\circ} \mathrm{C}$ (heating rate of $10^{\circ} \mathrm{C} / \mathrm{min}$ )

\section{HDO of phenol: catalytic experiments}

The catalytic experiments were performed in a $160 \mathrm{~mL}$ stainless steel PARR autoclave. Two laboratory lines, $\mathrm{N}_{2}$ and $\mathrm{H}_{2}$ lines were used with regulatory high-pressure valves. In a $160 \mathrm{~mL}$ autoclave, $75 \mathrm{~mL}$ of phenol solution $(0.150 \mathrm{~mol} / \mathrm{L})$, dissolved in decalin, was introduced. The catalyst $(100 \mathrm{mg})$ was then added and the autoclave was sealed. Afterward the system was purged 10 min with a stream of nitrogen, and then heated up to the desired temperature $\left(200^{\circ} \mathrm{C}\right)$. Once the desired temperature had been reached, 40 bars of hydrogen were introduced and the mixture was stirred at $650 \mathrm{rpm}$ for $2 \mathrm{~h}$. The hydrogen pressure is readjusted continuously during the catalytic tests (to compensate the slow decrease of pressure as $\mathrm{H}_{2}$ is consumed). Once the test was finished, hydrogen was slowly vented off after cooling down the system to room temperature. The system was finally purged for $10 \mathrm{~min}$ with a stream of nitrogen. The solution was filtered out and the catalyst was washed with acetone. The filtrate was then analyzed by GC (Agilent Technologies 6890N, column: BPX70, detector: FID $270^{\circ} \mathrm{C}$, column programming temperature: initial temperature $=60^{\circ} \mathrm{C} ;$ ramp $1=2^{\circ} \mathrm{C} / \mathrm{min}$ up to $70^{\circ} \mathrm{C}$; then $70^{\circ} \mathrm{C}$ for 2 minutes ; ramp $2=20^{\circ} \mathrm{C} / \mathrm{min}$ up to $250{ }^{\circ} \mathrm{C}$ ).

\section{Acknowledgements}

We acknowledge the FRS-FNRS, Fédération Wallonie-Bruxelles, Loterie Nationale, and Université catholique de Louvain for funding. We are also grateful to Jean-François Statsijns for technical assistance and François Devred for XRD and $\mathrm{NH}_{3}$-TPD measurements. We also thank the Applied Science inc. firm (USA) for carbon nanofibers (CNF). Financial support from the University of East Anglia through fully funded Ph.D. studentship for A.L.M. is gratefully acknowledged. Authors acknowledge the Faculty of Science Analytical Research Facility (NMR platform).

Keywords: lignin - hydrodeoxygenation • aluminosilicate • mesoporous nanofibers $\cdot$ phenol

J. N. Chheda, G. W. Huber, J. A. Dumesic, Angew. Chemie - Int. Ed. 2007, 46, 7164-7183

[2] J. Zakzeski, P. C. A. Bruijnincx, A. L. Jongerius, B. M. Weckhuysen, Chem. Rev. 2010, 110, 3552-3599.

[3] S. N. Naik, V. V Goud, P. K. Rout, A. K. Dalai, Renew. Sustain. Energy Rev. 2010, 14, 578-597.

[4] D. Mohan, C. U. Pittman, P. H. Steele, Energy \& Fuels 2006, 20, 848-889.

[5] S. P. S. Chundawat, G. T. Beckham, M. E. Himmel, B. E. Dale, Annu. Rev. Chem. Biomol. Eng. 2011, 2, 121-145.

[6] H. Ahmed, S. Nizamuddin, M. T. H. Siddiqui, S. Riaz, A. Sattar, D. K. Dumbre, N. M. Mubarak, M. P. Srinivasan, G. J. Gri, J. Environ. Chem. Eng. 2018, 6, 5101-5118.

[7] N. Arun, R. V Sharma, A. K. Dalai, Renew. Sustain. Energy Rev. 2015, 48, 240-255.

[8] X. Ma, K. Sakanishi, I. Mochida, Ind. Eng. Chem. Res. 1994, 33, 218-222.

[9] R. Prins, Adv. Catal. 2001, 46, 399-464

[10] X. Li, X. Luo, Y. Jin, J. Li, H. Zhang, A. Zhang, J. Xie, Renew. Sustain. Energy Rev. 2018, 82, 3762-3797.

[11] B. Güvenatam, O. Kurşun, E. H. J. Heeres, E. A. Pidko, E. J. M. Hensen, Catal. Today 2014, 233, 83-91.

[12] J. Wildschut, F. H. Mahfud, R. H. Venderbosch, H. J. Heeres, Ind Eng. Chem. Res. 2009, 48, 10324-10334.

[13] A. Gutierrez, R. K. Kaila, M. L. Honkela, R. Slioor, A. O. I. Krause Catal. Today 2009, 147, 239-246.

[14] T. Nimmanwudipong, R. C. Runnebaum, D. E. Block, B. C. Gates, Energy Fuels 2011, 25, 3417-3427.

[15] R. C. Runnebaum, R. J. Lobo-lapidus, T. Nimmanwudipong, D. E. Block, B. C. Gates, Energy Fuels 2011, 25, 4776-4785.

[16] C. Zhao, Y. Kou, A. A. Lemonidou, X. Li, J. A. Lercher, Angew. 
Chem. Int. Ed. 2009, 48, 3987-3990.

[17]

A. Roldugina, E. R. Naranov, A. L. Maximov, E. A. Karakhanov, Appl. Catal. A, Gen. 2018, 553, 24-35.

[18] D. D. Laskar, M. P. Tucker, X. Chen, G. L. Helms, B. Yang, Green Chem. 2014, 16, 897-910.

[19] J. A. Hunns, M. Arroyo, A. F. Lee, J. M. Escola, D. Serrano, K Wilson, Catal. Sci. Technol. 2016, 6, 2560-2564. W. Zhang, J. Chen, R. Liu, S. Wang, L. Chen, K. Li, ACS Sustain. Chem. Eng. 2014, 2, 683-691.

[21] C. Zhao, J. He, A. A. Lemonidou, X. Li, J. A. Lercher, J. Catal. 2011, 280, 8-16.

[22] Y.-C. Lin, C.-L. Li, H.-P. Wan, H.-T. Lee, C.-F. Liu, Energy Fuels 2011, 25, 890-896.

K. Kon, T. Toyao, W. Onodera, T. Toyao, W. Onodera, S. M. A. H. Siddiki, K. Shimizu, ChemCatChem 2017, 9, 2822-2827.

[24] P. Bhanja, K. Ghosh, S. S. Islam, S. M. Islam, A. Bhaumik, ACS Omega 2018, 3, 7639-7647.

[25] R. W. Jenkins, C. M. Moore, T. A. Semelsberger, A. D. Sutton, ChemCatChem 2017, 9, 2807-2815.

[26] S. Mondal, R. Singuru, S. C. Shit, T. Hayashi, S. Irle, Y. Hijikata, J. Mondal, A. Bhaumik, ACS Sustain. Chem. Eng. 2018, 6, 16101619.

P. M. De Souza, R. C. Rabelo-neto, L. E. P. Borges, G. Jacobs, B H. Davis, D. E. Resasco, F. B. Noronha, J. Am. Chem. Soc. 2017, 7, 2058-2073.

[28] M. Shetty, K. Murugappan, T. Prasomsri, W. H. Green, Y. Románleshkov, J. Catal. 2015, 331, 86-97.

[29] M.-A. Päivi, M. Dmitry Yu., Catalysts 2017, 7

[30] Y. Zeng, Z. Wang, W. Lin, W. Song, J. M. Christensen, A. D. Jensen, Catal. Commun. 2016, 82, 46-49. H. Xiong, H. N. Pham, A. K. Datye, Green Chem. 2014, 16, 46274643.

[32] Q. Li, Z. Wu, B. Tu, S. Soo, C. Ha, D. Zhao, Microporous Mesoporous Mater. 2010, 135, 95-104.

[33] Y. Li, W. Zhang, L. Zhang, Q. Yang, Z. Wei, Z. Feng, C. Li, J. Phys. Chem. B 2004, 108, 9739-9744.

[34] Y. Yue, A. Gédéon, J. Bonardet, N. Melosh, J. D. Espinose, R. Cambridge, U. K. June, A. August, Chem. Commun. 1999, 19671968. S. Wu, Y. Han, Y. Zou, J. Song, L. Zhao, Y. Di, Chem. Mater. 2004, 16, 486-492.

[36] Y. Xia, R. Mokaya, Microporous Mesoporous Mater. 2004, 68, 1-10.

[37] Y. Xia, R. Mokaya, Microporous Mesoporous Mater. 2004, 74, 179188.

[38] S. Zeng, Microporous Mesoporous Mater. 2005, 85, 297-304.

[39] Y. Li, Q. Yang, J. Yang, C. Li, Microporous Mesoporous Mater. 2006, 91, 85-91.

[40] J. C. Serrano-Ruiz, J. A. Dumesic, Energy Environ. Sci. 2011, 4, 83-99.

[41] C. Li, X. Zhao, A. Wang, G. W. Huber, T. Zhang, Chem. Rev. 2015, 115, 11559-11624.

[42] L. Cao, I. K. M. Yu, Y. Liu, X. Ruan, D. C. W. Tsang, A. J. Hunt, Bioresour. Technol. 2018, 269, 465-475.

[43] V. Kumar, D. Duc, J. Dharmaraja, S. Shobana, Bioresour. Technol. 2019, 271, 462-472.
X. Y. Yang, A. Vantomme, F. S. Xiao, B. L. Su, Catal. Today 2007,

128, 123-128.

[45] S. Cabrera, E. Haskouri, S. Mendioroz, C. Guillem, J. Latorre, D. Beltrán-porter, M. Dolores, P. Amorós, Chem. Commun. 1999, 1679-1680.

[46] Y. Li, Q. Yang, J. Yang, C. Li, J Porous Mater 2006, 13, 187-193.

[47] J. Klinowski, 1991.

[48] J. Rocha, J. Klinowski, J. Chem. Soc. Chem. Commun. 1991, 1, 1121-1122.

[49] T. V Choudhary, C. B. Phillips, Appl. Catal. A, Gen. 2011, 397, 112.

[50] T. Cordero-lanzac, R. Palos, I. Hita, J. M. Arandes, Appl. Catal. B Environ. 2018, 239, 513-524.

[51] J. M. Kim, R. Ryoo, Bull. Korean Chem. Soc 1996, 17, 66-68.

[52] H. Yang, G. Zhang, X. Hong, Y. Zhu, Microporous Mesoporous Mater. 2004, 68, 119-125.

[53] H. L. Castricum, M. C. Mittelmeijer-hazeleger, A. Sah, Microporous Mesoporous Mater. 2006, 88, 63-71.

[54] S. I. Karpov, F. Roessner, V. F. Selemenev, N. A. Belanova, O. O. Krizhanovskaya, Russ. J. Phys. Chem. A 2013, 87, 1888-1894.

[55] M. M. L. Ribeiro Carrott, F. L. Conceiçao, J. . Lopes, P. J. . Carrott, C. Bernardes, J. Rocha, F. Ramoa Ribeiro, Microporous Mesoporous Mater. 2006, 92, 270-285.

[56] A. P. Russo, M. M. L. Ribeiro Carrott, P. J. M. Carrott, Colloids Surfaces A Physicochem. Eng. Asp. 2007, 310, 9-19.

[57] A. M. Robinson, J. E. Hensley, J. W. Medlin, ACS Catal. 2016, 6, 5026-5043.

[58] T. Haynes, V. Dubois, S. Hermans, Appl. Catal. A, Gen. 2017, 542, 47-54.

[59] E. Furimsky, Appl. Catal. A Gen. 2000, 199, 147-190.

[60] D. Trine M.H., S. Magnus Zingler, H. Martin, J. Peter Arendt, G. Jan-Dierk, G. Jostein, M. Peter M., J. Anker Degn, Prog. Energy Combust. Sci. 2018, 68, 268-309.

[61] D. Hong, S. J. Miller, K. Agrawal, C. W. Jones, ChemComm 2010, 46, 1038-1040.

[62] S. Echeandia, B. Pawelec, V. L. Barrio, P. L. Arias, J. F. Cambra, C V Loricera, J. L. G. Fierro, Fuel 2014, 117, 1061-1073.

[63] S. Shao, H. Zhang, R. Xiao, X. Li, Y. Cai, Energy Convers. Manag. 2018, 155, 175-181.

[64] T. Haynes, O. Ersen, V. Dubois, D. Desmecht, K. Nakagawa, S. Hermans, Appl. Catal. B Environ. 2019, 241, 196-204.

[65] C. Pezzotta, G. Fleury, M. Soetens, S. Van Der Perre, J. F. M. Denayer, O. Riant, E. M. Gaigneaux, J. Catal. 2018, 359, 198-211. 


\section{Entry for the Table of Contents}

\section{Layout 1:}

\section{FULL PAPER}

Mesoporous acidic aluminosilicate nanofibers were prepared by a dual hard/soft templating methodology. A bifunctional catalyst was prepared by $\mathrm{Pd}$ deposition that gave complete conversion and up to $95 \%$ selectivity to cyclohexane in phenol HDO. This reaction is relevant to lignin valorisation. The acidic material also showed high hydrothermal stability and was recyclable without losing its initial fibrous structure.

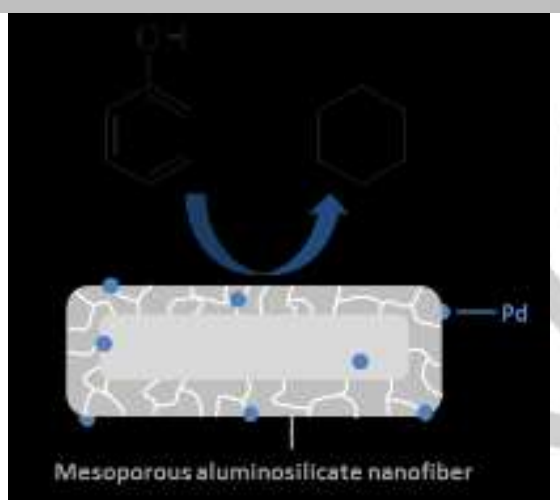

Tommy Haynes ${ }^{[a]}$, Thibaut D'hondt ${ }^{[b]}$, Alexander L. Morritt ${ }^{[c]}$, Yaroslav Z. Khimyak $^{[c]}$, Didier Desmecht ${ }^{[b]}$, Vincent Dubois $^{[b]}$ and Sophie Hermans ${ }^{*[a]}$

Page No. - Page No.

Mesoporous aluminosilicate nanofibers with a low Si/Al ratio as acidic catalyst for hydrodeoxygenation of phenol 